\title{
Synchronization of feedback-induced chaos in semiconductor lasers by optical injection
}

\author{
Atsushi Murakami ${ }^{1}$ and Junji Ohtsubo ${ }^{2}$ \\ ${ }^{1}$ Department of Electronic Engineering, The University of Electro-Communications, 1-5-1 Chofu-gaoka, Chofu-shi, \\ Tokyo 182-8585, Japan \\ ${ }^{2}$ Faculty of Engineering, Shizuoka University, 3-5-1 Johoku, Hamamatsu 432-8561, Japan
}

(Received 16 April 2001; revised manuscript received 9 November 2001; published 27 February 2002)

\begin{abstract}
In this paper, synchronization of chaotic oscillations in semiconductor lasers by optical injection in a transmitter-receiver configuration is studied numerically. A chaotic signal is generated from a semiconductor laser with optical feedback in the transmitter and is injected into the receiver laser without optical feedback. We examined the conditions of chaotic synchronization in the system. As a result, we observed complete chaos synchronization within a finite area of very small parameter mismatch between the two laser systems. In addition, chaotic oscillation synchronized by amplification phenomena was observed in the ordinary injectionlocking regime with higher optical injection ratios. We demonstrated that synchronization of chaotic oscillations by amplification results from a kind of injection locking under chaotic light injection and is very tolerant to the parameter mismatch between the two lasers, in contrast to the complete synchronization case.
\end{abstract}

DOI: 10.1103/PhysRevA.65.033826

PACS number(s): 42.55.Px, 42.65.Sf, 05.45.Xt

\section{INTRODUCTION}

Semiconductor lasers subjected to optical feedback from an external mirror or optical injection from another laser are known as nonlinear optical systems that can generate chaotic intensity fluctuations. A wide variety of nonlinear dynamical phenomena has aroused much interest in the field of laser chaos [1-8]. In particular, semiconductor lasers with optical feedback are good candidates as source generators for secure communications using chaos because they can generate highdimensional chaos due to feedback, ensuring a high level of security $[9,10]$. In secure communications, there is a problem of how the chaotic carrier wave form generated by a transmitter system can be reproduced in a receiver system.

In 1990, Pecora and Carroll first proposed the principle of chaos synchronization to produce the same chaotic wave forms in two coupled systems [11]. In this method, the transmitter system generating the chaotic wave form is composed of two subsystems, while the receiver system is a replica of one of the two subsystems. Coupling one variable of the transmitter system into the receiver system produces the same chaotic system on the receiver side. They demonstrated that a receiver's wave form converges into a chaotic trajectory of the transmitter system if all the conditional Lyapunov exponents in the receiver system are negative.

Several theoretical and experimental investigations of chaos synchronization and secure communications have been performed by applying the synchronization method in semiconductor lasers with optical feedback [12-21]. A typical configuration used in the investigations is shown in Fig. 1. The two lasers are coupled by unidirectionally injecting the transmitter laser's output into the receiver laser via an optical isolator (IS). In the two coupled systems, the transmitter system is composed of a semiconductor laser and an external mirror, emitting chaotic intensity fluctuation. The receiver system is composed of another semiconductor laser with optical injection. The rate equations for the coupled lasers are written as follows $[14,18]$ :

$$
\begin{aligned}
\frac{d E_{T}(t)}{d t}= & \frac{1}{2}(1+i \alpha)\left\{g\left[N_{T}(t)-N_{0}\right]-\gamma_{c}\right\} E_{T}(t) \\
& +\kappa_{\mathrm{ext}} E_{T}(t-\tau) \exp \left(-i \omega_{T} \tau\right) \\
\frac{d E_{R}(t)}{d t}= & \frac{1}{2}(1+i \alpha)\left\{g\left[N_{R}(t)-N_{0}\right]-\gamma_{c}\right\} E_{R}(t) \\
& +\kappa_{\mathrm{inj}} E_{T}\left(t-\tau_{c}\right) \exp \left\{-i\left[\Delta \omega\left(t-\tau_{c}\right)+\phi_{c}\right]\right\} \\
\frac{d N_{T, R}(t)}{d t}= & J_{T, R}-\gamma_{N} N_{T, R}(t)-g\left[N_{T, R}(t)-N_{0}\right]\left|E_{T, R}(t)\right|^{2}
\end{aligned}
$$

where $E$ and $N$ are the complex electric field and carrier number in the laser cavity, respectively, and the subscripts $T$

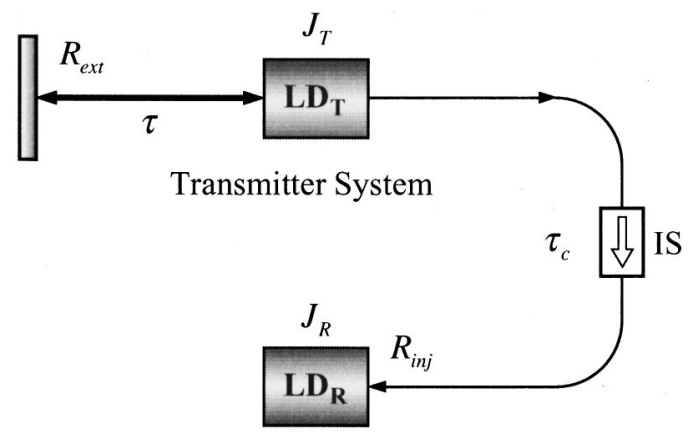

Receiver System

FIG. 1. Configuration for chaos synchronization in coupled semiconductor lasers. Chaotic output emitted from the transmitter laser $\left(L_{T}\right)$ with optical feedback is unidirectionally injected into the receiver laser $\left(L_{R}\right)$ via an optical isolator $(I S) ; J_{T}$ and $J_{R}$ represent the pump currents of the transmitter and receiver lasers, $\tau$ and $\tau_{c}$ represent the time delay for round-trip within the external cavity and for light propagation, and $R_{\text {ext }}$ and $R_{\text {inj }}$ represent the optical feedback rate and injection rate. 
and $R$ represent transmitter and receiver systems. $\alpha=3$ is the linewidth enhancement factor, $g=7.0 \times 10^{-6} \mathrm{~ns}^{-1}$ is the linear gain coefficient, $\gamma_{c}=518.9 \mathrm{~ns}^{-1}$ is the cavity decay rate, $\gamma_{N}=0.5 \mathrm{~ns}^{-1}$ is the spontaneous carrier decay rate, $J$ is the pump current normalized by electron charge, and $N_{0}=1.68$ $\times 10^{8}$ is the carrier number at transparency. $\tau$ is the roundtrip delay time within the external cavity, and $\tau_{t}$ is the propagation time of emitted light from the transmitter to receiver system. Feedback coefficient $\kappa_{\text {ext }}$ is given by $1 / \tau_{\text {in }}(1$ $\left.-R_{0}\right) \sqrt{R_{\text {ext }} / R_{0}}$, where $\tau_{\text {in }}=8$ ps is the round-trip time in the laser cavity, and $R_{0}$ and $R_{\text {ext }}$ represent intensity reflectivity of the laser exit facet and external mirror. $\omega_{T} \tau$ is the round-trip phase shift induced by light propagation within the external cavity, and $\omega_{T}$ is the angular frequency of the free-running transmitter laser. The coupling strength between the two systems can be given by an injection coefficient $\kappa_{\text {inj }}$ written as $1 / \tau_{\text {in }}\left(1-R_{0}\right) \sqrt{R_{\text {inj }} / R_{0}}$ in which $R_{\text {inj }}$ represents the percentage of the transmitter's output intensity injected into the receiver laser cavity. $\Delta \omega=\omega_{T}-\omega_{R}$ accounts for frequency detuning between the two free-running lasers, and $\phi_{c}$ is the phase difference between the two lasers. Here, we neglect the propagation delay time, i.e., $\tau_{c}=0$, for simplicity. From these equations, the necessary condition for realizing complete chaos synchronization are easily found to be

$$
\begin{gathered}
\kappa_{\mathrm{ext}}=\kappa_{\mathrm{inj}}, \\
\Delta \omega=0, \\
\phi_{c}=\omega_{T} \tau .
\end{gathered}
$$

Under these necessary conditions, the two systems can be described by the same, or equivalently the same, rate equations, and one may obtain a synchronous solution as $E_{R}(t$ $-\tau)=E_{T}(t)$. This is somewhat strange, in that the response of the receiver exceeds the effect of the transmitter with time lag $\tau$, the receiver anticipates the future state of the transmitter. This kind of synchronization with time lag $\tau$ is known as "anticipating synchronization" and can often be seen in synchronized time-delay systems $[9,10]$. The recent works have already predicted generation of anticipating synchronization in a different scheme based on coupled semiconductor lasers, in which the receiver laser has another external feedback $[14,19,20]$. In the works, in order to establish a symmetric condition for achieving anticipating chaos synchronization, the external feedback rate and the injection rate on the receiver side were adjusted so that total amount of the optical injection into the receiver laser corresponds to the transmitter's optical feedback. This symmetric condition is the same as our case when the receiver's feedback rate is set to be zero. Therefore, our configuration is a special simple case in considering chaos synchronization in coupled semiconductor lasers. Reference [14] has also indicated this point.

Contrary to the theoretical prediction, most of the experiments demonstrated that no time lag existed between the chaotic wave forms in the transmitter and receiver systems $[16,17]$. We believe that the experimental synchronization has a different origin than the principle of complete chaos synchronization. This paper discusses reproduction of the
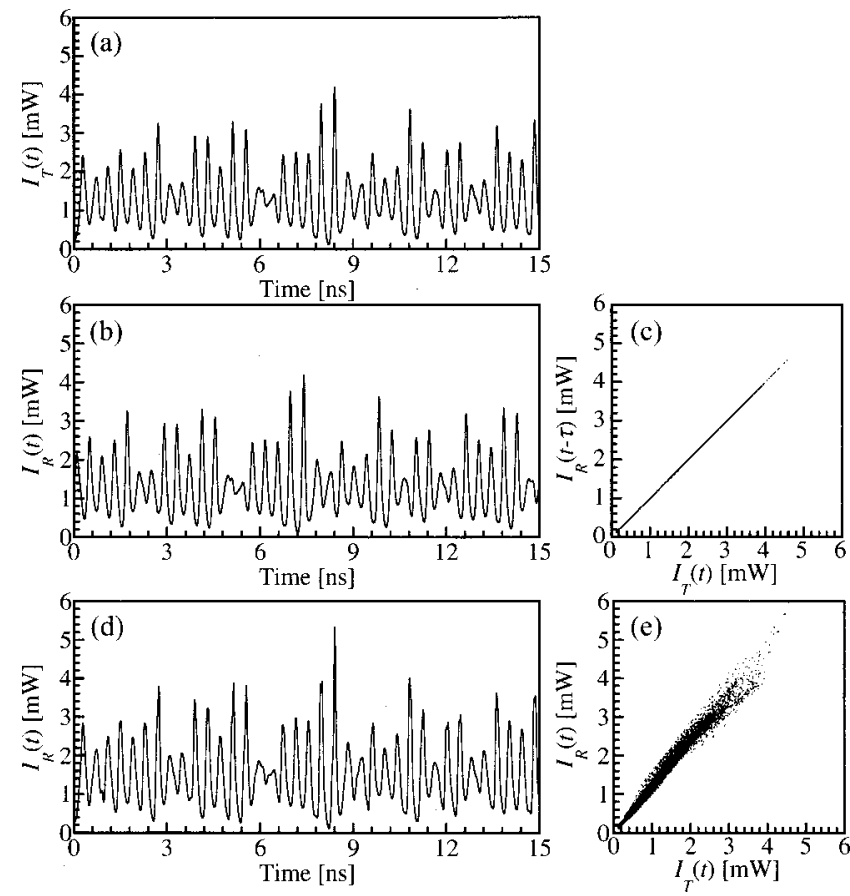

FIG. 2. Examples of reproduction of chaotic wave forms without frequency detuning. (a) Temporal chaotic wave form of transmitter laser (complete chaos synchronization) at $J_{T}=1.3 J_{\text {th }}, \tau$ $=1 \mathrm{~ns}$, and $R_{\mathrm{ext}}=0.015 \%\left(\kappa_{\mathrm{ext}}=1.96 \mathrm{~ns}^{-1}\right)$. (b),(c) Chaotic wave form in the receiver laser with the time lag $\tau$ at $R_{\text {inj }}=0.015 \%$ $\left(\kappa_{\mathrm{inj}}=1.96 \mathrm{~ns}^{-1}\right)$ and correlation plot between $I_{T}(t)$ and $I_{R}(t-\tau)$ obtained under the synchronization condition. (d), (e) Chaotic wave forms without any time lag in the receiver laser (synchronization of chaotic oscillations by nonlinear amplification) at a much increased injection rate of $R_{\mathrm{inj}}=22 \% \quad\left(\kappa_{\mathrm{inj}}=74.9 \mathrm{~ns}^{-1}\right)$ and corresponding correlation plot between $I_{T}(t)$ and $I_{R}(t)$.

feedback-induced chaos in coupled semiconductor lasers with and without the time lag by systematically investigating the model and the rate equations. As a synchronized chaotic state, we chose a continuous chaotic wave form due to destabilized relaxation oscillation caused in a weak optical feedback regime. Noted that it is not LFF chaos with coherence collapse caused by strong optical feedback $[14,19]$. Assuming the complex electric field as $E(t)=E_{0}(t) \exp [i \phi(t)]$, we can obtain a new set of rate equations for the amplitude, phase, and carrier number for each laser. We numerically simulated the rate equations employing the fourth-order Runge-Kutta algorithm. The semiconductor laser we consider here is a typical $\mathrm{Al}_{x} \mathrm{Ga}_{1-x} \mathrm{As}$ semiconductor laser operating at an optical wavelength of $800 \mathrm{~nm}$. In order to mainly treat deterministic phenomena, we neglect Langevin noise and other noise terms in the rate equations. The following sections will present the numerical results and discuss them. We will deal with the noise effect on synchronization in Sec. III C.

\section{SYNCHRONIZATION OF CHAOTIC OSCILLATIONS}

First, we present examples of reproduced chaotic wave forms without frequency detuning between the two lasers in Fig. 2. The device parameters of the two lasers are assumed 
to be the same. With increasing optical feedback, the transmitter laser can exhibit routes to chaos, which can be characterized by an excitation of the relaxation oscillation and the external cavity frequencies and by their mixing with the inherent nonlinearity of semiconductor lasers [4]. Figure 2(a) shows a transmitted chaotic wave form from the transmitter laser at $J_{T}=1.3 J_{\text {th }}, \quad \tau=1 \mathrm{~ns}, \quad$ and $R_{\text {ext }}=0.015 \%\left(\kappa_{\text {ext }}\right.$ $\left.=1.96 \mathrm{~ns}^{-1}\right)$. Under this condition, the chaotic wave form is continuous just after going through a quasiperiodic bifurcation and oscillates in the gigahertz range due to the destabilized relaxation oscillation. We applied a false nearestneighbor algorithm to this chaotic wave form and investigated the embedding dimension. As a result, this chaotic behavior was found to exhibit more than seven dynamical degrees of freedom, ensuring that the feedback-induced chaos is not low dimensional [25]. The output intensity emitted from the exit facet of the semiconductor laser is calculated as $I=\left[h c \omega \alpha_{m} /\left(4 \pi \mu_{g}\right)\right] E^{2}$, where $h$ is the Planck constant, $\alpha_{m}$ is the facet loss, and $\mu_{g}$ is the group refractive index [12]. From Eq. (4), we can find the necessary condition for complete chaos synchronization, under which the receiver completely synchronizes to the transmitter to be $R_{\text {inj }}$ $=0.015 \%\left(\kappa_{\mathrm{inj}}=1.96 \mathrm{~ns}^{-1}\right), \Delta \omega=0 \mathrm{GHz}$, and $\phi_{c}=\omega_{T} \tau$. The round-trip phase shift is $\omega_{T} \tau=2 n \pi$ ( $n$ being an integer) in this case. A receiver's output calculated under this condition is shown in Fig. 2(b). It is explicitly shown that the receiver synchronizes to the transmitter's chaotic wave form with time lag $\tau(=1 \mathrm{~ns})$, corresponding to the theoretical prediction. In Fig. 2(c), the correlation plot between $I_{T}(t)$ and $I_{R}(t-\tau)$ demonstrates that good synchronization is established. For a strong injection from the transmitter into the receiver, we can see a similar but slightly different chaotic output. Figure 2(d) presents the temporal wave form of the receiver at a much higher injection rate of $R_{\text {inj }}=22 \%\left(\kappa_{\text {inj }}\right.$ $=74.9 \mathrm{~ns}^{-1}$ ). The frequency detuning is also set to zero. The receiver produces a chaotic wave form similar to that in the transmitter, however, there is no time lag for synchronization in this case. It seems that the receiver just follows the injected intensity fluctuation. We also notice that the amplitude of fluctuation of the receiver's output became larger than that of the transmitter, which seems to be a nonlinear amplification phenomenon. The immediate response of the receiver output is a typical feature of this kind of nonlinear amplification phenomena in semiconductor lasers compared to the complete chaos synchronization case. Figure 2(e) shows the corresponding correlation plot between $I_{T}(t)$ and $I_{R}(t)$. The correlation is a little less than that in Fig. 2(c), but, similarity is good.

We will now describe another difference in the carrier. Figure 3 presents the orbit in a phase space of the intensity and the carrier number. Figures 3(a) and 3(b) show chaotic attractors, which correspond to the temporal wave forms of Figs. 2(b) and 2(d), respectively. The vertical axis represents carrier number normalized by the threshold carrier number $N_{\text {th }}$ for free-running operation. In the complete chaos synchronization, as shown in Fig. 3(a), the chaotic orbit was found to be identical to that in the transmitter. However, Fig. 3(b), the amplification case, shows a different orbit. We noticed that the carrier number becomes much lower than $N_{\text {th }}$
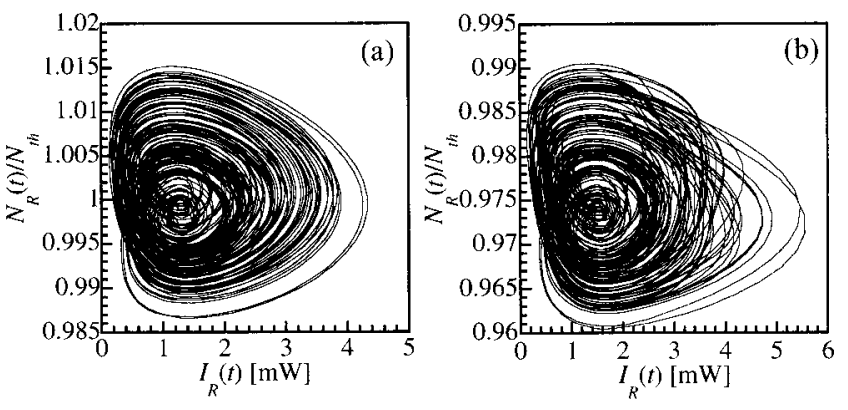

FIG. 3. Chaotic attractors consisting of intensity and carrier density in the receiver laser. (a) and (b) correspond to the temporal wave forms of Figs. 2(b) and 2(d), respectively.

in this case. It can easily be understood that the strong optical injection $\left(R_{\mathrm{inj}}=22 \%\right)$ leads to the threshold-carrier reduction in the receiver laser, since a sufficient number of photons for reaching the laser threshold is provided by the externally injected light. We actually confirmed in the other numerical results that the carrier reduction caused by the strong injection increases the amplification gain and enhances the synchronization effect related to similarity of the receiver output.

\section{PARAMETER MISMATCH BETWEEN TWO SYSTEMS}

Next, we investigate how parameter variation between the two systems influences the two types of chaos synchronization with and without the time lag, namely, complete chaos synchronization and chaotic oscillation synchronized by nonlinear amplification. As the variable parameters, we can consider external parameters that we can adjust in real experiments or internal laser parameters determined in manufacturing the lasers. In order to quantitatively estimate the similarity between two laser outputs, we introduce a synchronization error, which is defined as $\sigma=\langle| S_{T}$ $-S_{R}|\rangle /\left\langle S_{R}\right\rangle$, where $\langle\cdots\rangle$ denotes time average, and $S_{T}$ and $S_{R}$ represent normalized intensities of the transmitter and receiver lasers [18].

\section{A. Variation in external parameters}

First, we chose the injection rate $R_{\text {inj }}$ and the frequency detuning $\Delta f(=\Delta \omega / 2 \pi)$ as external parameters. We vary these parameters from those for the complete synchronization or amplification state in Figs. 2(b) and 2(d). The results are shown in Fig. 4, which presents a phase diagram as function of $\Delta f$ and $R_{\text {inj }}$. The boundaries denoted by the solid curves in the diagram represent a well-known injectionlocking regime that was analytically obtained by applying linear-stability analysis to the rate equations for semiconductor lasers with continuous wave (cw) light injection that corresponds to Fig. 1 in the absence of optical feedback in the transmitter system $[2,7]$. In the regime "unstable," various dynamical states are induced by the cw light injection, such as periodic (period 1, period 2, and so on), quasiperiodic, and chaotic states, but we do not show them to avoid congestion [6]. Figure 4(a) shows the result for complete chaos synchronization. We used a gray scale corresponding to each range 

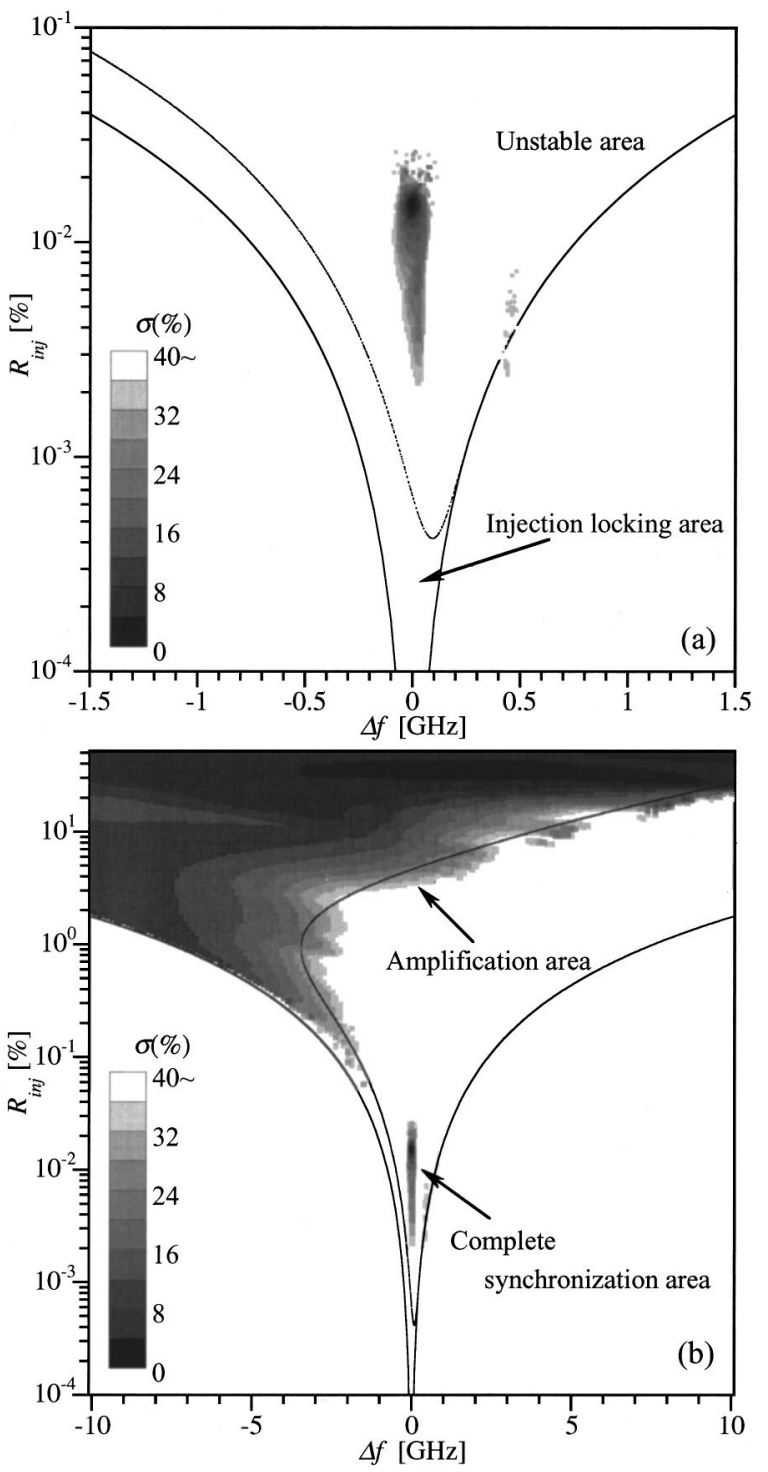

FIG. 4. Calculated synchronization error for variation in the frequency detuning $\Delta f$ and the injection rate $R_{\text {inj }}$. Quality of synchronization or amplification is represented by using gray scale with synchronization error. (a) and (b) correspond to the complete chaos synchronization of Fig. 2(b) and the amplification of Fig. 2(d), respectively. The boundary denoted by the solid line represents an injection-locking area for constant-intensity injection into the receiver laser. Coincidence between the amplification area and the injection-locking area is found in (b). (a) is also included in (b).

in the synchronization error to present the quality of synchronization clearly. We found a very small region for good synchronization near the condition of Eq. (4). The variation of the gray level reveals that varying the parameters from the synchronization condition decreases the quality of synchronization. Complete chaos synchronization is very sensitive, even to small variations in the parameter values. In contrast, we observed chaotic oscillations synchronized by amplification over a very wide area in a strong injection region of the phase space in Fig. 4(b). Furthermore, we found that the amplification regime overlaps with the injection-locking regime. The quality of amplification tends to increase with
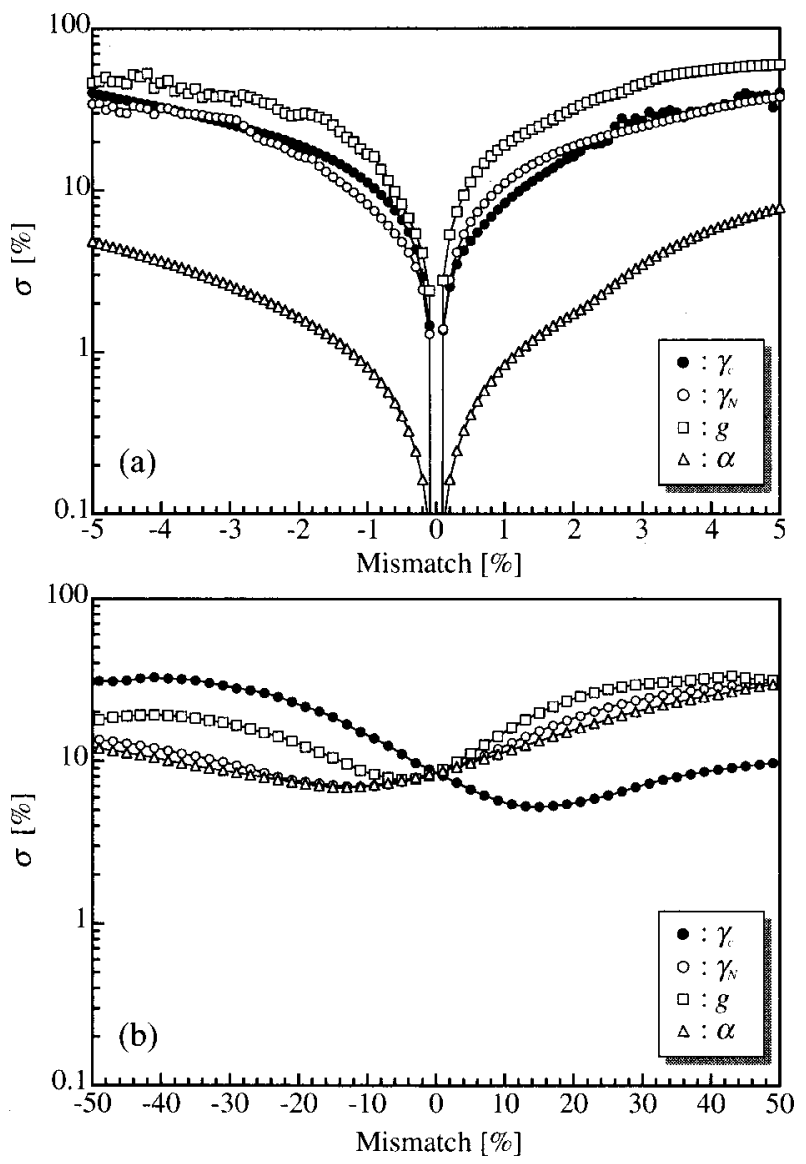

FIG. 5. Calculated synchronization error as a function of the parameter mismatch for $\gamma_{C}, \gamma_{N}, g$, and $\alpha$. (a) and (b) correspond to the complete chaos synchronization in Fig. 2(b) and nonlinear amplification in Fig. 2(d), respectively.

increasing injection rate and negative detuning. The complete synchronization area of Fig. 4(a) is included in Fig. 4(b). These two regimes are entirely separate from each other.

\section{B. Mismatch of internal laser parameters}

In practice, it is impossible to have two semiconductor lasers in which all the internal parameters are the same. Therefore, it is very important to investigate the influence of the mismatch of the two lasers on the quality of synchronization or amplification. The typical internal laser parameters we chose here are the cavity decay rate $\gamma$, the carrier decay rate $\gamma_{N}$, the linear gain coefficient $g$, and the linewidth enhancement factor $\alpha$. The result for complete chaos synchronization is shown in Fig. 5(a). The original synchronization state is the same as in Fig. 2(b). We also found sensitivity of the quality of synchronization to the parameter mismatch, the same as for the external parameter variations. The synchronization is lost even for a few percent of mismatch on each parameter. The $g$ mismatch is most effective in destroying synchronization, and the parameter $\alpha$ mismatch has much less effect on the quality of synchronization. In comparison, the influence of parameter mismatch on chaotic oscillations synchronized by amplification differs considerably. The re- 
sult is shown in Fig. 5(b). The original amplification state is the same as shown in Fig. 2(d). We note that the synchronization error is not reduced in amplification for parameter mismatch variation. It is very interesting that the matching is not the best condition for obtaining much more accurate amplification. The amplified chaotic oscillation is robust for variations in the mismatch of each parameter. In particular, the positive mismatch of cavity decay rate $\gamma_{c}$ or the negative mismatch of other parameters tends to yield better quality of amplification. A similar tendency in mismatching was observed for different conditions of the injection, i.e., for different values of the injection rate and the frequency detuning. Therefore, this may be an essential characteristic in semiconductor lasers exhibiting chaotic oscillation synchronized by amplification.

\section{Noise effect on synchronization}

Semiconductor lasers usually operate with their intrinsic noise that can affect significantly the chaotic dynamics. Therefore, we should investigate chaos synchronization property under the noise. We perform a numerical simulation including typical Langevin noise term $F_{i}(t)$ in Eqs. (1)-(3), which is modeled as a white Gaussian noise with zero mean and its autocorrelation written by $\left\langle F_{i}(t) F_{j}(t+\tau)\right\rangle$ $=D_{i j} \delta(\tau)$. The diffusion coefficients $D_{i j}\left(i, j: E_{0}, \phi\right.$, and $\left.N\right)$ are given by

$$
\begin{gathered}
D_{E_{0} E_{0}}=R_{\mathrm{sp}}, \\
D_{\phi \phi}=\frac{R_{\mathrm{sp}}}{E_{\mathrm{st}}^{2}}, \\
D_{N N}=R_{\mathrm{sp}} E_{\mathrm{st}}^{2}+\gamma_{N} N_{\mathrm{st}},
\end{gathered}
$$

where $E_{\mathrm{st}}$ and $N_{\mathrm{st}}$ are steady state values for the electric field amplitude and the carrier number, and $R_{\mathrm{sp}}$ represents a spontaneous emission rate given by $R_{\mathrm{sp}}=C_{\mathrm{sp}} \gamma_{N} N_{\mathrm{st}}$ in which we chose $C_{\mathrm{sp}}=10^{-7}$. We present the numerical result in Fig. 6 . The circle shows degree of synchronization with the noise as a function of the mismatch on the cavity decay rate for the complete synchronization case [Fig. 6(a)] and the amplification case [Fig. 6(b)]. To clarify comparison, we also present the result without the noise by the solid line, which corresponds to Fig. 5. It is clear that the noise easily degrades synchronization quality in the complete synchronization case. The synchronization error reaches several percent even under the matching condition. It results that the synchronous state in complete chaos synchronization is very unstable against noise. In contrast, the noise has much less effect on the quality of synchronization in the amplification case, shown in Fig. 6(b). Moreover, we found that the noise effect partly improves the degree of synchronization compared to that without the noise. The contrast of the noise effect on the two synchronization cases is interesting. We infer that the less effect of noise on the amplification case results from the noise reduction characteristics of injection-locked semiconductor lasers with strong injection [6].
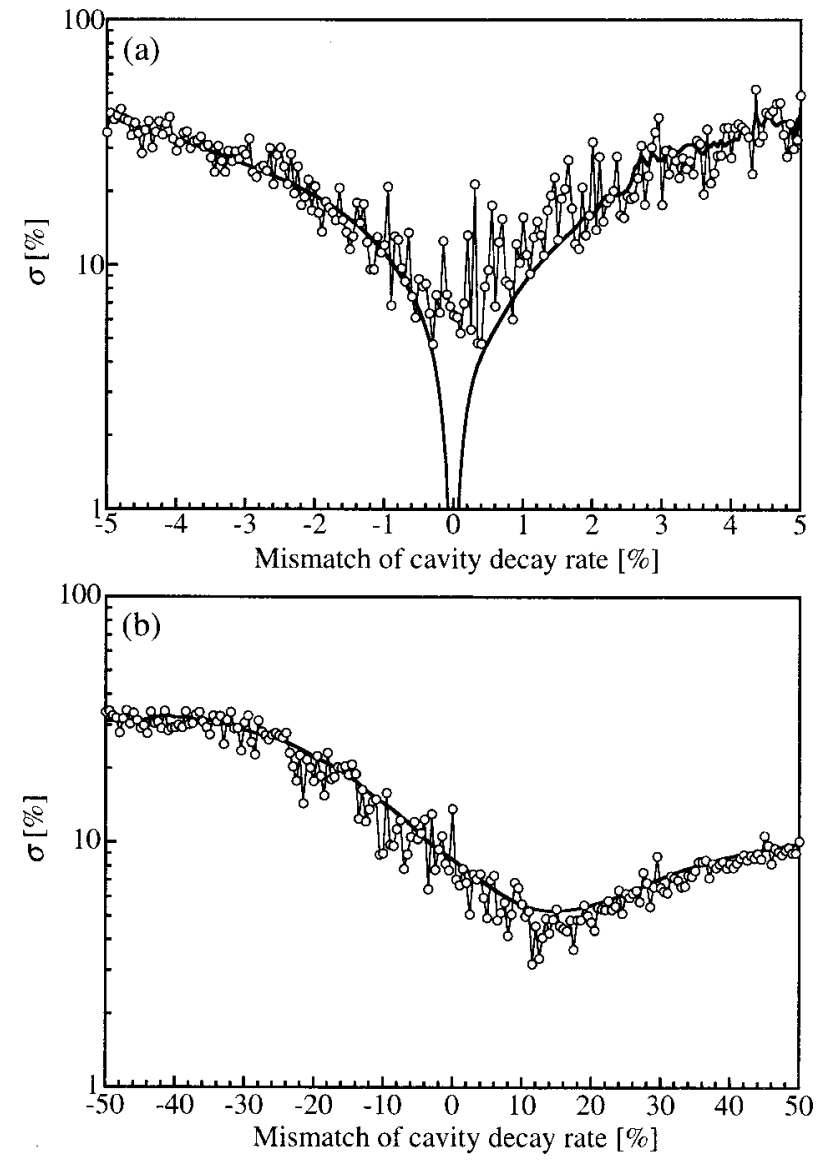

FIG. 6. Calculated synchronization error as a function of the mismatch on $\gamma_{c}$ with the Langevin noise included in the rate equations. The circle and the solid line represent the results with and without the noise, respectively.

\section{DISCUSSION}

In this section, we concentrate on chaotic oscillation synchronized by nonlinear amplification and discuss the numerical results. We previously presented some aspects for such synchronization. These are (i) the carrier reduction caused by strong injection enhances the amplification effect and increases the amplification gain and (ii) the synchronization regime overlaps with the injection-locking regime. We next perform a stationary analysis for the conventional injectionlocked semiconductor lasers to discuss these two results qualitatively. When the transmitter has no feedback, the injected term in Eq. (2) can be written as $E_{T}(t)$ $=E_{T, \mathrm{st}} \exp \left(i \phi_{T, \mathrm{st}}\right)$, where $E_{T, \mathrm{st}}$ and $\phi_{T, \mathrm{st}}$ represent the stationary amplitude and phase of the transmitter laser. If the pump current values of the two lasers are equal, $E_{T, \text { st }}$ $\cong E_{R, \text { st }}$ can be established. Under these conditions, we can obtain stationary solutions for the receiver laser from Eqs. (2) and (3), as follows:

$$
\begin{gathered}
E_{R, \mathrm{st}}^{2}=\frac{J_{R}-\gamma_{N} N_{R, \mathrm{st}}}{\gamma_{c}-2 \kappa_{\mathrm{inj}} \cos \Delta \phi_{\mathrm{st}}}, \\
N_{R, \mathrm{st}}=N_{0}+\frac{\gamma_{c}-2 \kappa_{\mathrm{inj}} \cos \Delta \phi_{\mathrm{st}}}{g} .
\end{gathered}
$$




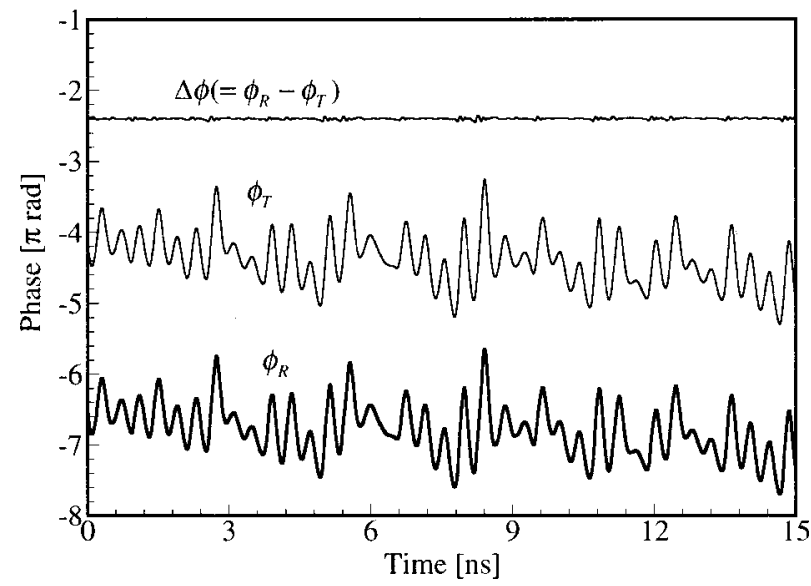

FIG. 7. Temporal fluctuations of the transmitter's phase $\left(\phi_{T}\right)$ and receiver's phase $\left(\phi_{R}\right)$, and their difference $(\Delta \phi)$ in the amplification case. The receiver's phase follows exactly the transmitter's phase fluctuation so that the phase difference between them is constant.

$$
\Delta \phi_{\mathrm{st}}=\phi_{R, \mathrm{st}}-\phi_{T, \mathrm{st}}=\sin ^{-1}\left\{-\frac{\Delta \omega}{\kappa_{\mathrm{inj}} \sqrt{1+\alpha^{2}}}\right\}-\tan ^{-1} \alpha
$$

Analyzing these equations, we found that $N_{R \text {,st }}$ decreases and $E_{R \text {,st }}$ increases with the increased injection strength $\kappa_{\text {inj }}$ and negative detuning $-\Delta \omega$. The increase of $E_{R \text {,st }}$ means light amplification [22]. Therefore, the stationary analysis agrees with the numerical result of (i).

In the conventional injection-locking scheme, an injected laser begins to oscillate at the same optical frequency as that of the injecting laser, and the relative phase between the two laser beams is fixed to a constant value. Such "frequency locking" and "phase locking" are typical phenomena in injection-locked semiconductor lasers and are fully explained by Eq. (8), showing that a stationary phase difference between the transmitter and receiver lasers is time constant. In the chaotic transmitter laser, however, both the optical frequency and the phase fluctuate chaotically. How can we discuss these locking phenomena for the chaotic light injection? Figure 7 shows temporal phase fluctuations in the chaotic oscillations synchronized by amplification in the injection-locking regime, which correspond to the intensity waveforms of Figs. 2(a) and 2(d). $\phi_{T}$ and $\phi_{R}$ are the phase fluctuations of the transmitter and receiver, and $\Delta \phi$ is their difference. It is clear that the receiver exactly follows the transmitter phase fluctuation and phase locking occurs. The locked phase value in the figure is about $\Delta \phi=-2.39 \pi$ and is identical to the stationary value calculated from Eq. (8). (Note that phase difference $\Delta \phi$ must be $2 n \pi$, where $n$ is an integer, in complete chaos synchronization.) In general, optical spectra of lasers are related to phase fluctuations. In our case, since the transmitter laser is in incoherent state caused by the chaotic oscillation and the transmitter's spectrum must be broadened due to the phase fluctuations on the subnanosecond time scale, compared to the solitary laser linewidth (less than $100 \mathrm{MHz}$ range) [22-24]. However, the synchro- nized phase of the receiver means that the two lasers exhibit very similar spectral characteristics in both the center frequency and linewidth. We consider that this is also a kind of frequency locking. Figure 7 is the results with zero detuning, but we actually confirmed that such frequency and phase locking for chaotic light injection were usually observed in a good amplification area with frequency detuning of Fig. 4(b). Therefore, injection locking for chaotic light injection can be defined as a phenomenon in which an injected laser begins to oscillate at the center frequency of the injecting laser and the injected-laser phase begins to exactly follow the chaotic fluctuations of the injecting-laser phase so that the relative phase is fixed to the phase-locking value. The above discussions verify that the chaos synchronized by nonlinear-amplification phenomena results from the injection locking of semiconductor lasers. We also investigated the phase dynamics, including the Langevin noise terms in numerical simulation, but found no essential difference.

\section{CONCLUSION}

We examined synchronization of chaotic oscillations in semiconductor lasers by optical injection in a transmitterreceiver configuration. The transmitter consists of a semiconductor laser with optical feedback and the receiver consists of a solitary semiconductor laser. There are two synchronization schemes. One is complete chaos synchronization in which the rate equations of the two systems can be described completely in equivalent forms for internal and external parameters. In this case, a time lag corresponding to the feedback-induced delay time exists between the transmitter and receiver chaos. The other one is the synchronization of chaotic oscillations induced by nonlinear-amplification phenomena. This was observed in the strong optical injection regime, and the synchronized chaotic wave form has no such time lag.

The characteristics of such a nonlinear amplification differ considerably from those of complete chaos synchronization. We have shown that the chaos synchronized by amplification was robust for a large mismatch between the two systems, while the complete chaos synchronization was very sensitive to a slight mismatch. Symmetry is strictly required to realize the best complete synchronization when constructing two coupled systems. This fact indicates that much effort would be required to experimentally observe synchronized chaos with the time lag. However, in the amplification case, a better quality of synchronized chaotic wave form can be obtained in asymmetric systems, than in symmetric systems. This should make it easy for us to observe this kind of phenomena in real experiments, since it is not necessary to construct two symmetric systems or to use two identical lasers in this case. We also investigated an effect of laser noise on the synchronization degree by including Langevin noise terms in the rate equations. As a result, a significant degradation of the synchronization performance was found in the complete chaos synchronization, while the synchronization by amplification was robust in the presence of the noise.

We explored the physical cause of the synchronization of chaotic oscillations by amplification. As a result, we found 
that the carrier reduction in the receiver laser, which is caused by strong injection, can enhance the amplification gain and can lead to a better amplification quality. Furthermore, the amplification regime corresponded to the conventional injection-locking regime in semiconductor lasers. Investigating phase fluctuations of the amplified state revealed that phase locking also occurs for chaotic light injection. We analyzed these results by performing a stationary analysis on the conventional injection-locking case and found good agreement between the numerical and analytical results. In addition, we considered a kind of frequency locking under chaotic light injection. From these results, we conclude that the synchronized chaotic oscillation without a time lag is caused by nonlinear amplification due to the injection locking in the receiver laser. Note that this agrees well with the earlier experimental study in Ref. [17].

For future studies, we expect that not only complete chaos synchronization, but also nonlinear light amplification by in- jection locking will be useful for reproducing a chaotic wave form with high accuracy in secure communications using chaos in semiconductor lasers. However, since the chaos synchronization method by amplification has a different robustness for parameter mismatch compared to that of conventional chaos synchronization, a different definition of security would be needed in realizing secure communications by this method. It is also interesting how one can encode and decode message in the chaos synchronization by amplification. In the near future, the conventional message encoding methods should be examined, such as, amplitude modulation of chaotic output [12] and injection-current modulation [17].

\section{ACKNOWLEDGMENTS}

The authors would like to thank Professor K. Atsuki for his valuable support, and Dr. Y. Liu, Dr. I. Fisher, and H. Takahashi for several useful discussions and suggestions.
[1] R. Lang and K. Kobayashi, IEEE J. Quantum Electron. 16, 347 (1980).

[2] G. H. M. van Tarwijk and D. Lenstra, Quantum Semiclassic. Opt. 7, 87 (1995); G. H. M. van Tarwijk and G. P. Agrawal, Prog. Quantum Electron. 22, 43 (1998).

[3] C. Masoller and N. B. Abraham, Phys. Rev. A 57, 1313 (1998).

[4] J. Ohtsubo, Opt. Rev. 6, 1 (1999).

[5] R. Lang, IEEE J. Quantum Electron. 18, 976 (1982).

[6] V. Kovanis, A. Gavrielides, T. B. Simpson, and J. M. Liu, Appl. Phys. Lett. 67, 2780 (1995); T. B. Simpson, J. M. Liu, K. F. Huang, and K. Tai, Quantum Semiclassic. Opt. 9, 765 (1997).

[7] V. Annovazzi-Lodi, A. Scire, M. Sorel, and S. Donati, IEEE J. Quantum Electron. 34, 2350 (1998).

[8] B. Krauskopf, N. Tollenaar, and D. Lenstra, Opt. Commun. 156, 158 (1998).

[9] K. Pyragas, Phys. Rev. E 58, 3067 (1998).

[10] H. U. Voss, Phys. Rev. E 61, 5115 (2000).

[11] L. M. Pecora and T. L. Carroll, Phys. Rev. Lett. 64, 821 (1990); L. M. Pecora and T. L. Carroll, Phys. Rev. A 44, 2374 (1991).

[12] C. R. Mirasso, P. Colet, and P. Garcia-Fernandez, IEEE Photonics Technol. Lett. 8, 299 (1996); A. Sanchez-Diaz, C. R.
Mirasso, P. Colet, and P. Garcia-Fernandez, IEEE J. Quantum Electron. 35, 292 (1999).

[13] V. Annovazzi-Lodi, S. Donati, and A. Scire, IEEE J. Quantum Electron. 33, 1449 (1997).

[14] V. Ahlers, U. Parlitz, and W. Lauterborn, Phys. Rev. E 58, 7208 (1998).

[15] S. Sivaprakasam and K. A. Shore, Opt. Lett. 24, 1200 (1999); IEEE J. Quantum Electron. 36, 35 (2000).

[16] Y. Takiguchi, H. Fujino, and J. Ohtsubo, Opt. Lett. 24, 1570 (1999); H. Fujino and J. Ohtsubo, ibid. 25, 625 (2000).

[17] I. Fischer, Y. Liu, and P. Davis, Phys. Rev. A 62, 011801(R) (2000).

[18] Y. Liu, H. F. Chen, J. M. Liu, P. Davis, and T. Aida, Phys. Rev. A 63, 031802(R) (2001).

[19] C. Masoller, Phys. Rev. Lett. 86, 2782 (2001).

[20] A. Murakami and J. Ohtsubo, Phys. Rev. E 63, 066203 (2001).

[21] J. K. White and J. V. Moloney, Phys. Rev. A 59, 2422 (1999).

[22] F. Mogensen, H. Olesen, and G. Jacobsen, IEEE J. Quantum Electron. 21, 784 (1985).

[23] G. P. Agrawal, IEEE J. Quantum Electron. 20, 468 (1984).

[24] D. Lenstra, B. H. Verbeek, and A. J. Den Boef, IEEE J. Quantum Electron. 21, 674 (1985).

[25] M. B. Kennel, R. Brown, and H. D. I. Abarbanel, Phys. Rev. A 45, 3403 (1992). 